\title{
Is over-reporting a problem in mandatory communicable disease reporting? The taiwanese experience on Enterovirus 71
}

\author{
Tseng Chu-Chun ${ }^{1}$, Yang Che-Ming ${ }^{2}$ \\ ${ }^{1}$ Mackay Memorial Hospital, Taipei, Taiwan \\ ${ }^{2}$ School of Health Care Administration and Shuang Ho Hospital, Taipei Medical University, Taipei, Taiwan
}

\begin{abstract}
Introduction: In Taiwan, severe enteroviral infections must be reported to the government within 24 hours to ensure that severe enterovirus 71 (EV71) infections can be detected early. The objective of this research was to ascertain whether over-reporting is a problem in mandatory disease-reporting systems.

Methodology: A multiyear cross-sectional study methodology was applied based on secondary data analyses. Data from the national notifiable communicable disease surveillance system of Taiwan Centers for Disease Control were analyzed to assess the trends and factors influencing reporting accuracy.

Results: From July 1999 to December 2008, 2,611 cases of severe enteroviral infection were reported in Taiwan. Among these cases, 1,516 were confirmed to be EV71 cases, and the remaining 1,095 were confirmed to be non-EV71 infections. The overall accuracy rate was $58 \%$. The accuracy rate was $60 \%-70 \%$ higher during epidemics (2000-2002, 2005, and 2008) and high seasons than it was in other seasons. The accuracy rate was highest among medical centers and lowest among district hospitals.

Conclusions: The results indicated that reports are more accurate during high seasons and peak years than during other periods. This might be attributable to the adequate level of specific educational programs for professionals when more cases occur, which could facilitate identification. Based on experiences in Taiwan, optimal training can ensure that surveillance systems are not inundated by false-positive reports.
\end{abstract}

Key words: severe enteroviral infection; enterovirus 71 (EV71); notifiable disease; over-reporting.

J Infect Dev Ctries 2014; 8(10):1294-1300. doi:10.3855/jidc.4623

(Received 25 December 2013 - Accepted 28 May 2014)

Copyright (C) 2014 Chu-Chun et al. This is an open-access article distributed under the Creative Commons Attribution License, which permits unrestricted use, distribution, and reproduction in any medium, provided the original work is properly cited.

\section{Introduction}

In April 1998, an enterovirus 71 (EV71) epidemic occurred in Taiwan [1], and 78 children died from associated complications [2]. The Taiwan Centers for Disease Control (CDC) reported that the rate of mortality caused by severe cases ranged from $10.0 \%$ to $25.7 \%$ [2]. Hence, EV71 infection is considered a serious threat to Taiwanese children.

EV71 was first isolated in 1970 in California, United States. Major outbreaks of EV71 have been documented in 17 countries, and the disease can cause a pandemic condition; therefore, vigorous surveillance and additional preventive measures are necessary [34]. Enteroviral infection has been frequently observed in children presenting with a broad range of clinical manifestations [5-9]. Most of the patients are asymptomatic or have minor symptoms such as undifferentiated febrile illness, rashes, and gastroenteritis. Serious diseases include hand, foot, and mouth disease, herpangina, aseptic meningitis, neonatal sepsis, and myocarditis. Enterovirus infects all age groups, but the chances of infection for children are much higher than for adults $[8,9]$.

The Taiwan CDC reported that, since 1998, 83\% of enteroviral infections with severe complications have occurred in children younger than five years of age. Infants younger than one year accounted for approximately $30 \%$ of all cases, and children between one and four years accounted for $50 \%$ of cases. Worldwide, non-polio enteroviral infections primarily affect children younger than ten years of age. The majority of severe cases in Taiwan have occurred in children younger than two years of age [5-7]. Also, compared with female patients, male patients are more prone to contracting the disease [10]; however, the cause of this sex discrepancy remains unknown. Regarding complications, viral meningitis is the most frequently encountered after an enteroviral infection [11]. From 1950 to $1981,66.7 \%$ of viral meningitis cases in the United States were caused by enteroviral infections. Similar findings have been reported in South Africa, United Kingdom, and Finland [12,13]. 
Similar to poliovirus, more neurological complications have resulted from EV71 than from any other enterovirus [14].

Enteroviral outbreaks are typically severe, and there are no effective drugs or vaccinations currently available for treating EV71. Taiwan CDC elevated cases of severe enteroviral infection from category 3 to category 2 in their scheme for controlling communicable diseases in 2004. According to Taiwan's Communicable Disease Control Act [15], category 2 infections must be reported within 24 hours, and infected patients should be confined for isolated treatment when necessary. Attending physicians must report cases of severe enteroviral infection if patients satisfy one of the following two criteria: (1) the patient presents typical symptoms of hand, foot, and mouth disease or herpangina complicated with myoclonic jerks, cerebritis, acute flaccid paralysis, acute hepatitis, myocarditis, or cardiopulmonary failure; and (2) infants younger than three months of age exhibiting symptoms and signs of sepsis, myocarditis, hepatitis, cerebritis, thrombocytopenia, multiple organ failure, or similar conditions (after excluding common causes such as bacterial infections) [16].

Surveillance systems are critical components of core public health functions for controlling infectious diseases. Public health surveillance is defined as the systematic collection, analysis, and interpretation of data essential to planning, implementing, and evaluating public health practices, and is closely integrated with the timely dissemination of these data to responsible agents [17]. The timeliness of reporting from healthcare professionals varies among countries and regions. For example, toward the end of the twentieth century, only 35 (approximately $60 \%$ ) of the 58 diseases and conditions recommended for national reporting in the US were reported in more than $90 \%$ of states and territories, and only 8 (approximately 14\%) were reported in less than $75 \%$ of states and territories [18]. Therefore, surveillance is a crucial tool for disease control, although it might be difficult to implement because of problems such as over- and under-reporting.

This research was conducted to determine whether over-reporting is a problem in Taiwan's mandatory disease reporting system. Accordingly, we examined whether increases in false-positive reports occurred during peak years and high seasons and among lowlevel healthcare facilities.

\section{Methodology}

This research was a multiyear cross-sectional study based on secondary data analyses. Data from the Taiwan CDC's National Notifiable Communicable Disease Surveillance System Database was analyzed, and the trends and factors influencing the accuracy of reports were assessed. The accuracy of reported EV71 infection was used as a dependent variable, and the independent variables were hospital, patient, and disease characteristics.

The reported case was designated in the end to be confirmed or unconfirmed EV71 infection. To define EV71 infection, Taiwan CDC either referred the case to an ad hoc expert committee to make the decision based on clinical aspects, or proceeded to specific laboratory serology. Taiwan CDC-contracted laboratories can conduct viral detection by testing for viral cultures or nucleic acids in throat swabs and serum, as well as neutralizing antibody detection (specifically anti-EV71 IgM antibodies) in serum [1920]. Since 2002, laboratory test results have been considered the standard for confirming EV71 infection. This study examined reports of severe enteroviral infections that occurred between July 1999 and December 2008. The variables extracted from the database included patient demographics, symptoms and signs, hospital level, diagnostic method, and confirmation of EV71 infection.

SPSS version 19.0 was used to conduct the data analyses. In addition to computing the descriptive statistics, a Chi-square test and logistic regression were performed to test the hypotheses. Results were considered statistically significant when $\mathrm{p}<0.05$.

Once this study was approved by Taipei Medical University School of Health Care Administration in 2009 , the database was released to the research team by Taiwan CDC.

\section{Results}

After excluding cases in which data were missing, 2,611 cases of severe enteroviral infection were reported between July 1999 and December 2008. Among these cases, 1,516 were confirmed EV71 cases, and the remaining 1,095 were non-EV71 cases. The overall accuracy rate was $58 \%$. The annual trend (Figure 1) shows that the accuracy rate was higher during epidemics (i.e., 2000-2002, 2005, and 2008) than during non-epidemics (mean, 62.34\% versus $36.78 \%)$. 
Figure 1. The trend of numbers of reported cases and confirmed cases and accuracy rates from 1999 to 2008

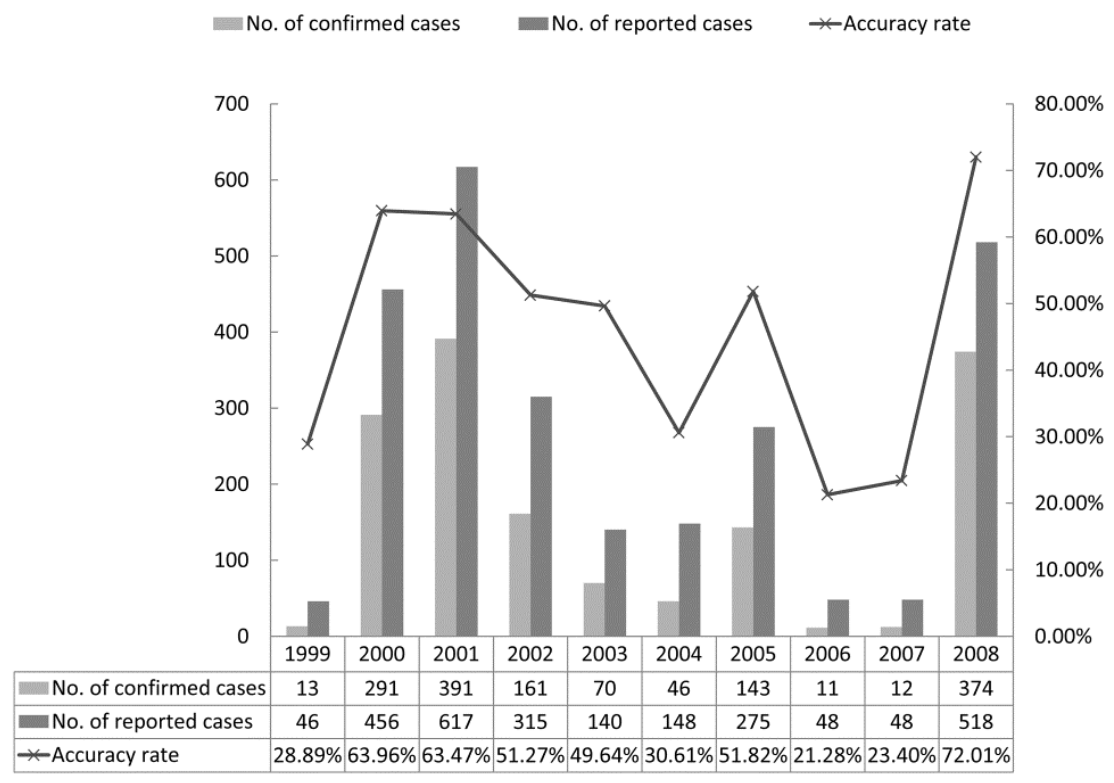

Table 1. Descriptive analysis results of patients reported with severe enteroviral infections

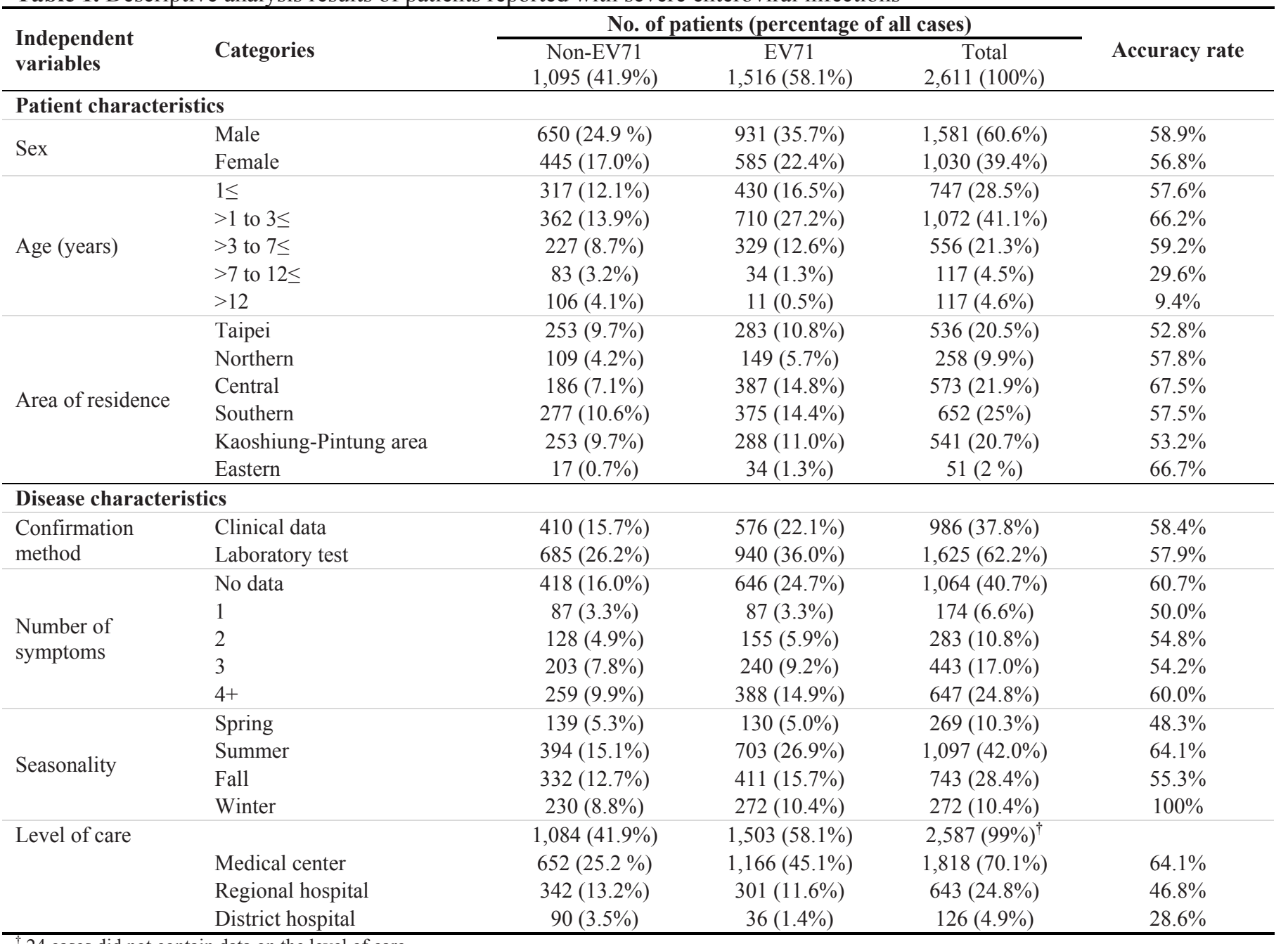


Table 1 shows that more male than female patients were reported to have EV71 and non-EV71 cases. The data indicated that 931 male $(35.7 \%)$ and 585 female patients $(22.4 \%)$ were confirmed to be EV-71 cases, and that 650 male $(24.9 \%)$ and 445 female patients $(17 \%)$ were confirmed to be non-EV-71 cases. Regarding age distribution, patients between one and three years of age constituted the largest group, followed by patients younger than one year. Patients between three and seven years of age constituted the third-largest group. More EV71 cases than non-EV71 cases involved patients younger than seven years of age. Conversely, more non-EV71 cases involved patients older than seven years.

Geographic area of residence was classified according to the system used for Taiwan's National Health Insurance program, which divides Taiwan into six regions (i.e., Taipei, Kaoshiung-Pintung, Northern, Eastern, Southern, and Western). According to the patients' area of residence, most EV71 cases involved patients living in the Central region, followed by those living in the Southern region. Most non-EV71 cases involved patients living in the Southern region.

Table 1 shows that $62.2 \%$ of all cases were confirmed by laboratory test results. Because of advancements in technology, laboratory tests have been used to confirm viral infections in Taiwan since 2002. Regarding the reported symptoms, no symptoms were recorded in the database for $40.7 \%$ of the total cases. Even for the EV71 cases, $42.6 \%$ of the records did not contain any symptom data; this can be attributed to the completeness (or lack thereof) of reports.

Spring, summer, fall, and winter were operationally defined as January-March, April-June, July-September, and October-December, respectively. After sorting the reports according to season, most cases were found to have been reported during summer (42\%), followed by fall (28.4\%).

Hospitals in Taiwan are categorized into three levels based on their accreditation (i.e., medical center, regional hospital, and district hospital), which is analogous to three levels of care; medical centers, regional hospitals and district hospitals provide tertiary, secondary, and primary care, respectively. The present research sample comprised 18 medical centers, 74 regional hospitals, and 249 district hospitals. Most cases were reported by medical centers

Table 2. Chi-square statistic results regarding the influence of disease and hospital characteristics on the accuracy rate

\begin{tabular}{|c|c|c|}
\hline \multirow{2}{*}{ Independent variables } & \multicolumn{2}{|c|}{ Accuracy rate } \\
\hline & $\chi^{2}$ & $\mathrm{P}$ value \\
\hline Sex & 1.120 & 0.292 \\
\hline Age & $178.747^{* * *}$ & 0.000 \\
\hline Level of care & $106.619^{* * *}$ & 0.000 \\
\hline Confirmation by laboratory test & 0.082 & 0.806 \\
\hline Presence of related symptoms & $9.838^{* * *}$ & 0.002 \\
\hline Reported during an epidemic & $96.914^{* * *}$ & 0.000 \\
\hline Seasonality & $32.208^{* * *}$ & 0.000 \\
\hline
\end{tabular}

$\mathrm{p}<0.1 ;{ }^{* *} \mathrm{p}<0.05 ;{ }^{* * *} \mathrm{p}<0.01$

Table 3. Logistic regression results of significant disease and hospital characteristics against EV71 confirmation status

\begin{tabular}{|c|c|c|c|c|}
\hline Variable & Category & Regression coefficient & OR & $95 \% \mathrm{CI}$ \\
\hline \multirow{4}{*}{ Age (years) } & $1 \leq^{\dagger}$ & & & \\
\hline & $>1$ to $3 \leq$ & 0.323 & $1.417^{* * *}$ & $1.154-1.741$ \\
\hline & $>3$ to $7 \leq$ & 0.062 & 1.064 & $0.843-1.343$ \\
\hline & $>12$ & -2.343 & $0.096^{* * *}$ & $0.052-0.176$ \\
\hline \multirow[b]{2}{*}{ Level of care } & Medical center & 1.448 & $3.559^{* * *}$ & $2.800-6.470$ \\
\hline & Regional hospital & 0.776 & $1.723^{* * *}$ & $1.359-3.385$ \\
\hline \multirow{4}{*}{ Seasonality } & Spring $^{\dagger}$ & & & \\
\hline & Summer & 0.599 & $1.821^{* * *}$ & $1.362-2.436$ \\
\hline & Fall & 0.220 & 1.246 & $0.921-1.685$ \\
\hline & Winter & 0.084 & 1.088 & $0.790-1.499$ \\
\hline
\end{tabular}

$\mathrm{p}<0.1 ;{ }^{* *} \mathrm{p}<0.05 ;{ }^{* * *} \mathrm{p}<0.01 ;{ }^{\dagger}$ reference groups 
(70.1\% of cases), followed by regional hospitals, and then district hospitals. Most EV71 cases were reported by medical centers, accounting for $77.6 \%$ of all confirmed cases.

Tables 2 and 3 show the effects of the independent variables on accuracy rates according to Chi-square test and logistic regression results, respectively. Regarding patient characteristics, the patients' ages and areas of residence had a significant impact on the accuracy rate of reports. The patients in the one-tothree-year age group were reported to have the highest accuracy rate. By contrast, those who were older than seven years of age were associated with the lowest accuracy rate. The effect of sex on the accuracy rate was not significant, although the presence of symptoms, number of symptoms, and seasonality exerted a significant effect on the accuracy rate. Moreover, the accuracy rate was significantly higher during summer and in peak years. In addition, the level of care (i.e., hospital level) had a significant effect on the accuracy rate. District hospitals exhibited the lowest accuracy rate.

\section{Discussion}

Compared with the findings of previous studies, we observed similar sex and age distribution in EV71 cases $[5-7,10]$. The effect of sex on the accuracy rate was not significant; however, age distribution and level of care were significantly correlated with accuracy. These results indicate that higher reporting volumes coincided with higher accuracy rates.

In our study, medical centers, which are responsible for tertiary care in Taiwan, exhibited the highest accuracy rates. Medical centers accounted for $70.1 \%$ of the reports in this study. Theoretically, primary care facilities should account for most reports because they are "gatekeepers" under most circumstances. However, tertiary care facilities in Taiwan reported more cases than did primary care facilities. Because no clearly established referral system exists among Taiwanese healthcare providers, people may seek care at any level of hospital. Generally, people visit medical centers when they feel severely ill. Consequently, medical center physicians are more likely to encounter patients with severe enteral infections who present related symptoms. Although our results indicated that doctors in highlevel hospitals are more experienced at diagnosing enteral infections than are doctors at hospitals of other levels, doctors in clinics and district hospitals are visited by patients presenting with ambiguous symptoms, which may contribute to the high number of false-positive cases reported at the primary care level; thus, we cannot infer that primary care physicians are less capable than their secondary- or tertiary-level counterparts in diagnosing enteral viral infections.

Communicable disease surveillance can be based on voluntary and mandatory reporting. Case reporting is a selective process involving multiple stages, the first of which requires a symptomatic person to present at a healthcare service provider; consequently, factors affecting the probability of people visiting a physician affect the characteristics of reported cases. The ensuing hurdles are whether healthcare providers can diagnose specific diseases and whether they are willing to report the case. In a mandatory reporting system, willingness is reinforced through coercion. To minimize the occurrence of physicians overlooking actual cases, health authorities encourage reporting by adopting positive or negative (or both) reinforcement strategies. Generally, interest in research on infectious disease surveillance systems that do not rely completely on mandatory reporting has grown; many health authorities have developed reporting requirements for laboratories and/or hospitals, especially for diseases that require specific laboratory results for confirmation [21].

Encouraging the reporting of infectious diseases is critical for the worldwide surveillance of infectious diseases, although countries might conceal outbreaks of infectious diseases to avoid economic loss [22]. For governments to be responsive to global surveillance, they must rely on their domestic reporting systems. However, over- and under-reporting of infectious diseases is an ongoing problem. For example, overreporting might occur when clinicians must submit initial reports to authorities, or when epidemics occur. This may be attributed to fear of punishment among healthcare professionals for failing to report cases. Additionally, over-reporting is preferable to underreporting from the perspective of timely control.

Previous research has indicated that health departments might be notified with reports on only $10 \%-25 \%$ of the actual number of cases [23]. From the perspective of preventing the spread of diseases, over-reporting is preferred to under-reporting. Because under-reporting leads to inadequate control measures, most authorities do not punish false-positive reports, though they do punish under-reporting.

However, certain costs are associated with overreporting. For example, field investigators and laboratory systems become overburdened when too many false alarms occur. When false-positive rates are 
excessive, surveillance systems become inefficient [22]. National reporting systems should be designed after considering both over- and under-reporting.

Currently, Taiwan's Communicable Disease Control Act defines five categories of communicable disease and stipulates that certain communicable diseases require mandatory reporting. Furthermore, when physicians detect communicable diseases or suspect them while diagnosing and treating patients, they must immediately implement necessary infection control measures and report the case(s) to their local health authorities. Depending on the category of a communicable disease, physicians must file a report within 24 hours (categories 1 and 2), one week (category 3), or in accordance with the regulations of relevant health authorities (categories 4 and 5).

Taiwan's communicable disease surveillance system has undergone considerable redevelopment during the past two decades, particularly following the outbreak of severe acute respiratory syndrome in 2003. The entire surveillance system has evolved from a single notifiable communicable disease surveillance system to an integrated network including sentinel and syndromic reporting systems. Gradually, surveillance duty has been delegated to physicians.

When reporting is mandatory, physicians typically over-report when they become aware of impending epidemics because they fear punishment for failing to report actual cases; consequently, false-positive rates surge. However, our study results indicated that the accuracy rate was higher during high seasons and peak years than it was in other years. This might be attributed to Taiwanese physicians' knowledge and experience with enteroviral infections. Most healthcare professionals are adequately trained in identifying diseases; hence, when more cases occur, it is easier for physicians to identify actual cases. By contrast, when few cases occur, a few mistakes have a greater effect on accuracy rates because the denominator (i.e., the number of overall cases) is smaller. In other words, when a mandatory reporting system is well established, the magnitude of an epidemic is not exaggerated; thus, authorities should have few concerns regarding a high number of false alarms. When adequate training is provided, mandatory reporting systems are not inundated by false alarms.

The findings of this research may be limited by the applied confirmation method. Taiwan's CDC currently recognizes two methods of confirmation. However, because laboratory methods have become increasingly favored, the number of cases confirmed by ad hoc committees has decreased accordingly. Nevertheless, the ad hoc committee method was the main tool used during the first three years of our study data, potentially affecting the validity of our findings. In addition, the generalizability of our findings is limited because healthcare delivery systems differ among countries.

\section{Conclusions}

The results of this study indicated that the accuracy rate of EV71 reports during peak years and high seasons was significantly higher when compared to other periods. The accuracy rate was also higher at the medical center level and lower at the district hospital level.

The argument against establishing mandatory reporting systems is based on the magnitude of overreporting resulting from fear of punishment for failing to report actual cases. However, our results indicated that the accuracy rate in Taiwan was higher during high seasons and peak years than it was in other years, which might be attributed to the experience and knowledge of Taiwanese physicians regarding enteroviral infections. Also, most healthcare professionals are adequately and periodically trained to identify communicable infectious diseases. Therefore, if a mandatory reporting system is well established, the magnitude of epidemics is not exaggerated during an epidemic. Finally, authorities and physicians need not be concerned about a high number of false alarms when the reporting of communicable diseases is mandatory. Periodic and adequate training should ensure that surveillance systems are not inundated by false-positive reports.

\section{Acknowledgements}

The authors acknowledge the assistance from the Taiwan $\mathrm{CDC}$ in providing the research data.

\section{Authors' contributions}

The authors contributed equally to this study.

\section{References}

1. Centers for Disease Control, R.O.C. Taiwan (2013) Enteroviruses infection with severe complications. Taipei City: CDC. Available: http://www2.cdc.gov.tw/public/Data/08101636171.pdf. Accessed 20 March 2013.

2. Centers for Disease Control, R.O.C. Taiwan (2013) Enteroviruses infection with severe complications. Taipei City: CDC. Available: http://www.cdc.gov.tw/professional. Accessed 20 March 2013.

3. Betty AB, Steven M (1999) Molecular Epidemiology and Evolution of Enterovirus Strains Isolated from 1970 to 1998. J Virol 73: 9969-9975. 
4. Oberste MS, Maher K, Kilpatrick DR (1999) Typing of human enteroviruses by partial sequencing of VP1. J Clin Microbiol 37: 1288.

5. Jenista JA, Powell KR, Menegus MA (1984) Epidemiology of neonatal enterovirus infection. J Pediatr 104: 685-690.

6. Prather SL, Jenista JA, Menegus MA (1984) The isolation of nonpolio enteroviruses from serum. Diagn Microbiol Infect Dis 2: $353-357$.

7. Rotbart HA (1995) Enteroviral infections of the central nervous system. Clin Infect Dis 20: 971-981.

8. Morens DM (1978) Enteroviral disease in early infancy. J Pediatr 92: 374-377.

9. Melnick JL (1996) My role in the discovery and classification of the enteroviruses. Annu Rev Microbiol 50: 1-24.

10. Gelfand HM, Holgium AH, Marchetti GE (1963) A continuing surveillance of enterovirus infections in healthy children in six United States cities. I. Viruses isolated during 1960 and 1961. Am J Hyg 78: 1963.

11. Selekman J (2003) Preventing meningitis. Pediatrics Nurs 29: 467-469.

12. Hsu CM (1995) Outbreak of aseptic meningitis in Taipei in spring. J Formos Med Assoc 94: 14-18.

13. Kao CH (2000) Outbreak of aseptic meningitis among adults in southern Taiwan. J Microbiol Immunol Infect 367: 192196.

14. Liu CC, Tseng HW, Wang SM, Wang JR, Su IJ (2000) An outbreak of enterovirus 71 infection in Taiwan, 1998: epidemiologic and clinical manifestations. J Clin Virol 17: 23-30.

15. Taiwan Department of Health (2009) Communicable Disease Control Act, Amended January 7th. Taipei: Government press.

16. Centers for Disease Control, R.O.C. Taiwan (2013) Enteroviruses infection with severe complications. Taipei City: CDC. Available: http://www.cdc.gov.tw/professional/diseaseinfo.aspx?treeid= beac $9 \mathrm{c} 103 \mathrm{df} 952 \mathrm{c} 4 \&$ nowtreeid $=6 \mathrm{~b} 7 \mathrm{f} 57$ aafde $15 f 54 \&$ tid $=9000$ 59B505FD76DF. Accessed 20 March 2013.
17. Jajosky RA, Groseclose SL (2004) Evaluation of reporting timeliness of public health surveillance systems for infectious diseases. BMC Public Health 4: 29.

18. Roush S, Birkhead G, Koo D, Cobb A, Fleming D (1999) Mandatory reporting of diseases and conditions by health care professionals and laboratories. JAMA 282: 164-170.

19. Centers for Disease Control, R.O.C. Taiwan (2013) The Manual for Infectious Specimen Collection. Taipei City: CDC.

Available: http://www.cdc.gov.tw/professional/infectionreportinfo.aspx? treeid $=56 \mathrm{ca} 56252 \mathrm{a} 0 \mathrm{fa} 705 \&$ nowtreeid $=\mathrm{ca} 20 \mathrm{fc} 0 \mathrm{dab} 36 \mathrm{f} 820 \& \mathrm{ti}$ $\mathrm{d}=$ BA473CA51877403D. Accessed 10 February 2013.

20. Centers for Disease Control, R.O.C. Taiwan (2013) Manual of Standard Operation Procedure of Communicable Diseases (II). Taipei City: CDC. Available: http://www.cdc.gov.tw/professional/infectionreportinfo.aspx? treeid $=56$ ca56252a0fa $705 \&$ nowtreeid $=2$ f29fde932b498b3\&ti d=DB3601024EC3BA60. Accessed 10 February 2013.

21. Chorba TL, Berkelman RL, Safford SK, Gibbs NP, Hull HF (1989) Mandatory reporting of infectious diseases by clinicians. JAMA 262: 3018-3026.

22. Cash RA, Narasimhan V (2000) Impediments to global surveillance of infectious diseases: consequences of open reporting in a global economy. Bull World Health Organ 78: 1358-1367.

23. Stephen BT, Keewhan C, Philip SB (1983) The Surveillance of Infectious Diseases. JAMA 249: 1181-1185.

\section{Corresponding author}

Yang Che-Ming

School of Health Care Administration and Shuang Ho Hospital

Taipei Medical University, Taipei, Taiwan

Phone: +886-2-27361661 ext. 3616

Fax: +886-2-23789788

Email: cyang@tmu.edu.tw

Conflict of interests: No conflict of interests is declared. 